\title{
Épistémologie et méthodologie de la recherche dans le secteur LANSAD : qu'apporterait une harmonisation des pratiques?
}

Jean-Paul Narcy-Combes et Marie-Françoise Narcy-Combes

\section{OpenEdition \\ Journals}

Édition électronique

URL : http://journals.openedition.org/asp/2136

DOI : 10.4000/asp.2136

ISBN : 978-2-8218-0382-4

ISSN : 2108-6354

Éditeur

Groupe d'étude et de recherche en anglais de spécialité

Édition imprimée

Date de publication : 31 décembre 2000

Pagination : 243-251

ISSN : 1246-8185

Référence électronique

Jean-Paul Narcy-Combes et Marie-Françoise Narcy-Combes, «Épistémologie et méthodologie de la recherche dans le secteur LANSAD : qu'apporterait une harmonisation des pratiques ? », ASp [En ligne], 27-30 | 2000, mis en ligne le 28 janvier 2011, consulté le 30 avril 2019. URL : http://

journals.openedition.org/asp/2136; DOI : 10.4000/asp.2136

Ce document a été généré automatiquement le 30 avril 2019

Tous droits réservés 


\title{
Épistémologie et méthodologie de la recherche dans le secteur LANSAD : qu'apporterait une harmonisation des pratiques?
}

\author{
Jean-Paul Narcy-Combes et Marie-Françoise Narcy-Combes
}

1 Cet article vise à faire partager à ses lecteurs une étape nouvelle, de formalisation, d'une réflexion engagée sur la théorisation de la pratique (Narcy 1995) et prolongée au cours de plusieurs années de travail dans le cadre d'un séminaire doctoral animé conjointement avec $\mathrm{F}$. Demaizière. Cette réflexion est, en ce sens, le fruit de nos échanges et, de manière considérable, l'œuvre collective du séminaire doctoral auquel nous participons.

\section{Justification du sujet}

2 Notre domaine s'est considérablement développé dans les dix dernières années, de nombreuses thèses ont été soutenues en particulier et l'un de nous deux a siégé à un grand nombre de jurys, tant dans notre secteur que dans d'autres. Les grandes disparités qui peuvent être notées aussi bien dans la construction épistémologique de ces thèses, que dans leur méthodologie et leur organisation, ont conduit notre séminaire doctoral à s'interroger profondément sur ce que ces différences suggéraient et sur les moyens de mieux se situer dans un ensemble assez disparate, reconnaissons-le.

3 Cette hétérogénéité, si elle reflète la richesse et le dynamisme du domaine, n'est pas sans inconvénient. Il est difficile d'évaluer les travaux et de se positionner. Certaines thèses sont des thèses parcours où une carrière transparaît, d'autres sont d'intéressantes bibliographies raisonnées, des compilations où le travail de terrain prend une place secondaire, d'autres enfin suivent directement des approches empruntées soit aux sciences humaines, soit plus directement aux sciences de l'éducation. Enfin, un certain nombre se situent clairement dans le champ théorique d'une école linguistique précise. 
Tout ceci est parfaitement acceptable. Il serait néanmoins satisfaisant également que le type de travail qui est présenté soit défini, avec ses points forts et les biais que ce choix induit. Ce n'est pas encore assez le cas, et les choix sont souvent présentés comme allant de soi.

5 Nous gagnerions en lisibilité interne et externe à réfléchir ensemble sur ce point, pas nécessairement pour unifier rigidement les pratiques, mais pour situer les travaux dans ces pratiques. Cela nous permettrait de mieux asseoir notre légitimité scientifique et de faire reconnaître à notre champ de réflexion l'expertise à laquelle il doit parvenir. Ainsi, par exemple, les décisions institutionnelles pourraient être prises en tenant compte des résultats de nos travaux, quand ces résultats sont en contradiction avec les représentations courantes ou qu'ils invalident plus ou moins nettement les croyances incantatoires du moment, ce qui n'est pas encore le cas. Enfin, les chercheurs novices seraient également plus à l'aise pour s'orienter.

Nous commencerons par un regard bibliographique qui nous montrera l'ampleur de la tâche pour les chercheurs de notre domaine. Nous préciserons ensuite ce que nous entendons exactement lorsque nous employons les termes du titre car, comme nous le verrons dans la partie suivante, l'observateur joue un rôle personnel important dans son observation, qui nécessite que l'on comprenne son acception des termes qu'il emploie et l'influence que son point de vue exerce sur sa recherche.

7 Nous présenterons ensuite un modèle de démarche de recherche spécifique à notre contexte, ce qui justifiera que nous passions aux implications pratiques de ce cadre. Enfin, nous aborderons la façon dont pourraient se gérer les relations avec les chercheurs des domaines qui collaborent avec le nôtre.

\section{Références bibliographiques et définitions}

8 Se tourner vers la bibliographie nous met en face de la complexité du travail d'un chercheur de notre domaine qui souhaite se situer clairement sur le plan épistémologique et méthodologique. Encore convient-il d'ajouter que dans notre cas, les références aux sciences du langage n'incluent que rarement la linguistique ou la linguistique appliquée sur le plan méthodologique ou épistémologique. Ce n'est pas sans conséquence, et il serait tout aussi sage de le faire, mais nous atteignons là nos limites...

9 Il n'est pas de notre propos de conseiller une approche de la recherche, disons simplement que nous pouvons nous interroger sur la validité d'une recherche qui, dans son organisation, économiserait les références à plusieurs des sciences de référence que nous avons citées. L'entreprise est ambitieuse et on peut concevoir qu'un ouvrage collectif de synthèse faciliterait grandement la tâche des chercheurs novices.

Le mot épistémologie est bien entendu polysémique; il englobe les méthodes propres à chaque science ou domaine scientifique, mais également les démarches de la pensée scientifique en général. Il peut se référer également au problème général de la vérité scientifique. Nous aimerions garder tous ces sens, dans une acception synthétique qui rappellerait que, pour nous, l'épistémologie serait la réflexion sur la construction et la gestion du savoir dans un domaine donné dans son rapport avec tous les autres domaines de la réflexion scientifique.

11 Nous reprendrons à J. Swales la notion que la méthodologie implique le respect des règles et des conventions de discours de la communauté scientifique dont on revendique 
l'appartenance. Ce qui imposera au secteur LANSAD de définir clairement ses règles et ses conventions de discours.

\section{Le chercheur dans sa recherche}

E. Morin a été l'un des premiers à affirmer qu'il était important de réintégrer l'observateur dans son observation et donc de mesurer les effets que son point de vue et son conditionnement avaient sur sa recherche (Narcy 1998a).

N. Elias (1973), lui, distingue l'engagement du chercheur, son implication personnelle dans ce qu'il entreprend, et sa distanciation, le recul que la méthodologie scientifique lui impose.

Des auteurs aussi différents que Damasio, Buser ou Varela confirment avec les observations matérielles que la neurophysiologie permet maintenant le rôle majeur de nos émotions dans la construction de notre pensée. Notre affectif explique notre cognition. Il convient donc que, à la suite de Kühn, pour qui l'objectivité était le respect des conventions et de la terminologie des chercheurs de notre domaine, nous allions jusqu'à admettre qu'objectif n'est pas l'antonyme de subjectif. Ces deux concepts se déclinent sur des continua.

La subjectivité est liée au point de vue et à l'affectif (à l'engagement), l'objectivité à la rigueur de l'adhésion méthodologique (la distanciation). Ce qui nous conduit à souligner le paradoxe suivant: il est plus objectif d'être très subjectif et de mesurer les effets de cette subjectivité que d'être peu subjectif et de confondre cela avec de l'objectivité.

Seliger et Shohamy (1989) nous rappellent qu'il est impératif de se positionner et ils nous donnent des outils pour le faire. Souhaitons qu'ils soient écoutés et que nous parvenions à adapter à notre contexte les outils qu'ils nous proposent. La richesse de notre domaine ne nous facilitera pas la tâche pour suivre ces propositions, et il est loin d'être simple pour nous de définir la recherche et son objet.

\section{La recherche et son objet}

\subsection{Inscription dans un champ théorique}

Pour Bourdieu (1979), la rupture épistémologique est le passage de l'objet réel - c'est-àdire, pour nous, l'objet tel qu'il apparaît à travers nos représentations façonnées par le milieu socioculturel dans lequel nous évoluons - à l'objet construit. Pour ce faire, il est nécessaire d'inscrire l'objet de recherche dans un champ théorique. Or le problème auquel se heurtent les chercheurs dans notre domaine, c'est l'absence de champ théorique construit. Il sera dès lors nécessaire d'en construire un pour chaque étude abordée, ce qui explique la présence de contextes théoriques en apparence surdimensionnés dans les thèses présentées en didactique des langues. Du fait qu'il n'existe pas de paradigme unifié, quelle lecture des théories va-t-on privilégier?

\subsection{Monothéisme, polythéisme}

18 Il existe en recherche deux grandes tendances dans l'approche épistémologique pour lesquelles David Block emploie la métaphore suivante. L'«approche monothéiste » 
favorise un paradigme unique. Pour les tenants du monothéisme, la vérité est unique, exclusive, absolue. Sa démarche vise à la simplification, à la rationalisation et à la généralisation. La recherche dans ce domaine est cumulative, c'est-à-dire que le nouveau savoir s'ajoute au savoir existant en couches successives.

Les approches polythéistes voient la vérité plurielle, diverse, relative telle qu'elle leur apparaît à travers le kaléidoscope des théories, variant les angles de vision. Leur domaine est celui de la complexité, et elles prennent en compte les représentations et les effets de contexte. La recherche ouvre des perspectives multiples, non exclusives les unes des autres.

Culturellement, la France est monothéiste. L'objectivité des auteurs de cette communication leur impose de dire qu'ils sont polythéistes, tout comme est polythéiste l'orientation de ce travail.

\subsection{Trois approches}

Trois approches découlent du polythéisme :

- Le syncrétisme, ou intégration non raisonnée et non justifiée de diverses théories.

- L'éclectisme, qui se justifie dans la pratique pour répondre à des problèmes de terrain, tant qu'il n'y a pas de réponse théorique unique, mais qui en recherche apparaît comme la simple acceptation du statu quo, et donc ne permet pas d'aller de l'avant.

- L'intégration raisonnée et cohérente des diverses théories lors d'une recherche donnée, en fonction de la question de recherche et d'un contexte particulier, pour conduire à une problématique qui elle-même sera validée ou invalidée en fin de parcours.

Notre position apparaît à la façon subjective dont nous présentons les trois approches possibles. Autant l'éclectisme s'impose dans la pratique. Il ne serait pas acceptable de renoncer à prendre des décisions sous prétexte qu'il n'y a pas de théories suffisamment validées pour le faire, autant il serait réducteur sur le plan de la recherche, car il serait une acceptation d'un statu quo ante qui ne correspond pas au désir du chercheur de mieux comprendre les choses.

\section{La démarche de recherche}

L'observation de multiples recherches nous amène à proposer le modèle suivant, qui, comme tout modèle, se veut intérimaire et souhaite expliciter sans être trop précis, puisque tout n'est pas encore clarifié.

Figure 1. Modèle

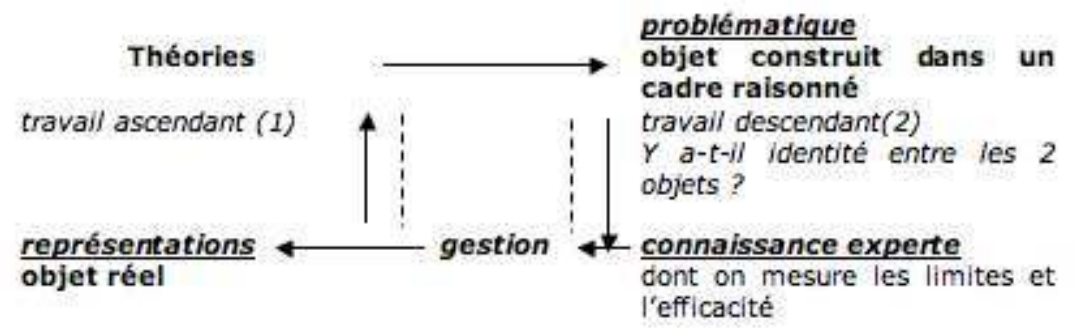

On relance une recherche dès que les limites gênent. 
Lesceil ascendant (1) n'est bien sûr pas isolé d'un travail descendant, dans la mesure où le chercheur ne saurait rencontrer l'objet réel sans que son regard ne soit modifié par les théories dont il a déjà une connaissance plus ou moins intuitive issue d'une expertise déjà partiellement en place, ou de représentations. Il en est de même du travail descendant (2), qui ne saurait être isolé d'un travail ascendant. Néanmoins, pour le premier (1), il s'agit d'aller de l'objet de recherche à un objet construit par le recueil de données observables et quantifiables pour déterminer des indicateurs qui permettront de construire des concepts isolés, puis systémiques. Ce travail implique une formation méthodologique rigoureuse au recueil de données. Ces données, ou les concepts qu'elles auront permis d'élaborer, seront confrontées aux concepts des diverses théories en amont afin de construire un objet cohérent et efficace (puisque, pour nous, la didactique est une praxéologie) qui permettra de répondre à la question de recherche.

Il arrive, comme le rappelait P. Doucet dans son intervention lors du débat qui a suivi la communication dont est issu cet article, que l'on aboutisse à quelque chose de différent de ce que l'on cherchait. Il faudrait alors s'assurer si nos représentations correspondaient vraiment à l'objet réel que nous recherchions. En d'autres mots, avions-nous posé la bonne question de recherche, celle qui nous interpellait vraiment?

Le travail descendant (2) a pour objet de s'assurer de la validité des hypothèses émises, ou de vérifier si l'objet construit correspond à l'objet réel. Il le fera si sa définition, et les décisions qu'elle permet, ne créent pas de dysfonctionnement.

\section{Implications pratiques}

\subsection{Le cadre théorique}

Dans ce contexte, le cadre théorique prend une place importante dans le document final, ce qui est logique quand on se rappelle le nombre de sciences de référence que le chercheur convoque pour mener à bien une recherche. La collaboration des équipes de recherche est nécessaire pour arriver à le réduire par références croisées avec les cadres théoriques de recherches se situant dans des contextes proches ou partageant une question de recherche de même ordre.

On peut pratiquer pour construire ce cadre ce que nous avons appelé la «technique des entonnoirs ». Il ne s'agit en aucun cas de concevoir cette étape comme une démonstration d'érudition, mais comme le reflet des choix du chercheur lorsqu'il rencontre une théorie qu'il juge opératoire dans son contexte. Les théories sont donc passées au filtre de la question de recherche, pour aboutir à une synthèse et à un positionnement du chercheur, selon ce qu'il hypothétise opératoire dans son contexte.

Cela impose une technique de rédaction quelque peu répétitive, car il est nécessaire de préciser après la présentation de chaque théorie ce qui justifie qu'on l'ait convoquée, et ce que l'on en retient pour l'expérience, pour quelle raison et dans quel but.

\subsection{La problématique}

29 La problématique est, en quelque sorte, la synthèse des synthèses et positionnements qui a pour objet de construire un modèle et/ou une métaphore et/ou un cadre théorique 
provisoire, dans le but d'aboutir à une redéfinition de la question de recherche et des hypothèses.

\section{L'expérimentation}

L'expérimentation implique un travail sur le terrain pour lequel il existe une logique des choix méthodologiques. Le recueil de données par des questionnaires, enquêtes, entretiens semi-directifs ou observation, l'analyse de ces données et leur interprétation font appel à des techniques rigoureuses qui ne seront pas développées ici. Des auteurs comme Benoît Ravelleau, Quivy et Van Campenhoudt, ou Juan ont publié à ce propos des travaux utiles au chercheur. La légitimité de notre domaine découlera de la rigueur avec laquelle nous manipulons ces outils.

\section{Les conclusions}

31 Le travail de recherche aboutit soit à une non invalidation des hypothèses après un travail déductif, soit après un travail inductif à une théorie, un modèle ou une métaphore qui deviendront à leur tour des outils.

Les résultats seront donnés avec une prise de distance qui implique de mesurer les biais induits par l'engagement du chercheur d'une part, les choix méthodologiques d'autre part, d'indiquer les conditions de réplication et d'indiquer les conditions d'invalidation.

Toute recherche nous conduit non seulement à préciser la validité de nos hypothèses, mais également à préciser la validité de notre cadre théorique. Cette validation des problématiques devrait contribuer progressivement à la construction d'un cadre théorique général moins diversifié, ce qui facilitera le travail des chercheurs et permettra de résoudre le problème des divergences théoriques qui nous interpelle aujourd'hui. Il s'agit là d'un objectif à long terme, et nous sommes loin de la constitution d'un paradigme unique pour notre domaine.

\section{Relations avec les chercheurs des autres domaines}

Ces quelques lignes ne sont que les prémisses d'une réflexion, et elles ne se voudraient en aucun cas polémiques.

La richesse et la complexité des études que nous avons à mener nous imposent de collaborer avec des chercheurs de domaines scientifiques voisins, dont certains se constituent en science. Il ne serait pas opportun d'accepter une position ancillaire. Leurs concepts et leurs résultats sont pour nous des outils, mais la spécificité de notre domaine, l'intervention sur le terrain, distingue souvent nos préoccupations des leurs.

Reconnaissons cependant que, si nous utilisons leurs outils, il serait préférable de respecter leurs normes d'emploi, et donc de les connaitre. Un exemple fréquent de ce problème est la confusion qui apparaît dans les travaux entre des résultats qu'on aurait obtenus par l'observation scientifique d'un comportement et ceux que nous a donnés le recueil des représentations des apprenants sur leurs comportements. Cet impératif montre que la formation des chercheurs novices gagnerait à se développer sur ce point. Veillons par contre à ce qu'un spécialiste d'un autre domaine n'attende pas d'un chercheur du nôtre qu'il soit aussi savant que lui dans son domaine. Il nous faudrait alors être trop savants !

Enfin, si l'on se tourne vers des chercheurs tels que Rod Ellis, on prend conscience de l'importance que revêt la validation pratique des modèles, ou théories. Valorisons donc le 
travail de terrain en assurant sa rigueur scientifique et en diffusant ses résultats de façon plus systématique, mais aussi plus convaincante. Ceci nécessite une collaboration plus étroite entre les chercheurs universitaires et les praticiens.

\section{Conclusion}

Ce rapide survol d'une question qui mérite plus d'attention nous amène à conclure que, pour le moment, le seul paradigme qui puisse unifier notre domaine serait d'ordre méthodologique. Il serait d'ailleurs probablement contre-productif d'essayer d'établir un paradigme théorique unifié. Si c'est le cas, nous gagnerions à approfondir notre réflexion commune, tant au niveau des chercheurs qu'à celui de la formation à la recherche. Nous gagnerions en légitimité ce que nous perdrions en liberté et, après les années passionnantes du débroussaillage, il peut être satisfaisant d'organiser le jardin. La question restera, sera-t-il à la française ou à l'anglaise?

\section{BIBLIOGRAPHIE}

Annoot, E. 1996. Les formateurs aux nouvelles technologies : le sens du changement. Paris: Ophrys.

Atlan, J. 1999. « Les différences individuelles en apprentissage de l'anglais et les nouvelles technologies éducatives : l'interaction entre aptitude et traitement pédagogique ». Thèse de doctorat de l'Université de Technologie de Compiègne.

Block, D. 1999. « Who framed SLA research? ». In Cameron, Lynne \& Graham Low, Researching and Applying Metaphor. Cambridge : Cambridge University Press.

Bourdieu, P. 1979. La distinction. Paris : Minuit.

Buser, P. 1998. Cerveau de soi, cerveau de l'autre. Paris : Odile Jacob.

Cosnier, J. 1994. La Psychologie des émotions et des sentiments. Paris : Retz.

CRAP. Cahiers pédagogiques 362 «À l'heure d'internet ». 1998. Paris : Cercle de recherche et d'action pédagogique.

Cyrulnik, B. 1997. L'ensorcellement du monde. Paris : Odile Jacob.

Damasio, A. R. 1999. Le sentiment même de soi. Paris : Odile Jacob.

Elias, N. 1973. La civilisation des mœurs. Paris : Calman Levy.

Ellis, R. 1997. SLA Research and Language Teaching. Oxford : Oxford University Press.

Gardner, H. 1996. Les intelligences multiples. Lyon : Retz.

Juan, S. 1999. Méthodes de recherche en sciences sociohumaines. Paris : Presses Universitaires de France.

Kuhn, T. 1970. The structure of Scientific Revolutions. Chicago : University of Chicago Press.

Laborit, H. 1994. La légende des comportements. Paris : Flammarion. 
Larsen-Freeman, D. et M. H. Long. 1991. An Introduction to Second Language Research. Harlow : Longman.

Linard, M. 1996. Des machines et des hommes. Paris : L'Harmattan.

Little, D. « Strategies, counselling and cultural difference: why do we need an anthropological understanding of learner autonomy? ». In Proceedings, Sixth Conference in Developing Autonomous Learning, Barcelona, 3-6 Septembre 1997.

Morin, E. 1977. La Méthode 1. «La nature de la nature ». Paris : Seuil.

Narcy, J.-P. 1995. « La théorisation de la pratique ». ASp 7-10, 443-452.

Narcy, J.-P. 1997. «Vers une pratique théorisée et humaniste ». In Ginet, A. (dir.), Du laboratoire de langues à la salle de cours multi-médias. Paris : Nathan, 45-90.

Narcy, J.-P. 1998a. «Le Monde à notre portée ». Études de linguistique appliquée 112, 435-448.

Narcy, J.-P. 1998b. «Représentations, apprentissages et supports multimédias ». Cahiers de l'Apliut 17/3, 14-24.

Quivy, R. et L. Van Campenhoudt. 1988. Manuel de recherche en sciences sociales. Paris : Dunod.

Pateau, J. 1994. « Approche comparative interculturelle : étude d'entreprises françaises et allemandes ». Thèse de doctorat : Université Paris X Nanterre.

Popper, K. R. 1969. Conjectures and refutations. Londres : Routledge \& Kegan Paul.

Raveleau, B. 1995. Cours de psychologie sociale. Angers : IPSA.

Seliger, H.W. et E. Shohamy. 1989. Second Language Research Methods. Oxford : Oxford University Press.

Sturge-Moore, O. 1997. « De la nécessaire inclusion du contexte culturel dans les cours d'anglais de spécialité ». Thèse de doctorat, Université Bordeaux 2.

Varela, F. J. 1886. Quel savoir pour l'éthique? (trad. de l'anglais). Paris : La Découverte.

\section{RÉSUMÉS}

Cet article a pour objectif de suggérer des conditions pour que les recherches du secteur aient une plus grande lisibilité tant pour les chercheurs du domaine que pour ceux des autres domaines. Il convient aussi d'accroitre notre légitimité scientifique par des pratiques rigoureuses. Une première conséquence est, à la suite d'E. Morin, de réintégrer l'observateur dans son observation et de prendre la mesure des biais que son point de vue et ses options introduisent dans les résultats. Un modèle du cycle de la recherche est présenté ; il prend en compte ce qui précède et une conception «polythéiste » des références aux diverses théories vers lesquelles nous nous tournons. Des implications pratiques sont mises en avant, en particulier en ce qui concerne la rédaction des thèses. Enfin, les relations avec les chercheurs des autres domaines de référence ont été abordées. Il s'agit de trouver la complémentarité des regards et d'éviter toute velléité d'ignorance de leurs critères ou toute tendance à œuvrer dans l'ancillarité.

The aim of this article is to offer suggestions to clarify research in order to make it more readable to researchers whether they belong to our field or not. Another objective is to develop a strict methodology to improve the scientific validity of our research. We think after Edgar Morin that we need first to take into account the researcher's role as the observer of the object observed, in 
order to be able to assess the implications of his or her own bias and decision making in the results obtained. This presentation proposes a model for the full development of research which is based on a "polytheist" approach of practical applications for researchers, especially those who need to write for the completion of their thesis, and finally reflections on what relations with researchers in related fields of research could be like, if we all want to raise our awareness of what our own criteria, and to avoid being mere followers.

\section{INDEX}

Keywords : complexity, epistemology, methodology, qualitative, research, validation

Mots-clés : complexité, épistémologie, méthodologie, qualitatif, recherche, validation

\section{AUTEURS}

\section{JEAN-PAUL NARCY-COMBES}

Jean-Paul Narcy-Combes est Professeur à l'Université de La Rochelle où il met en place une équipe de recherche sur les effets de l'intégration des technologies éducatives sur les institutions. Il est l'auteur de divers articles et également d'ouvrages tels que Apprendre une langue étrangère (1990). Jean-paul.narcy-combes@wanadoo.fr

\section{MARIE-FRANÇOISE NARCY-COMBES}

Marie-Françoise Narcy-Combes est actuellement en poste à l'IUFM des Pays de la Loire comme formatrice en didactique de l'anglais. Elle est engagée dans un projet de recherche sur le développement de la recherche action auprès des enseignants de terrain avec une petite équipe rattachée à l'équipe émergeante de l'Université de La Rochelle IDEA avec Jean-Paul NarcyCombes.mf.narcycombes@wanadoo.fr 